\title{
Enhanced CAE system for detection of exudates and diagnosis of diabetic retinopathy stages in fundus retinal images using soft computing techniques
}

\author{
Janaki Sathya $\mathrm{D}^{1, \mathrm{a}}$, Geetha $\mathrm{K}^{2}$ \\ ${ }^{1}$ Department of Electrical \& Electronics Engineering, PSG College of Technology, Coimbatore, India \\ ${ }^{2}$ Department of Electrical \& Electronics Engineering, JCT College of Engineering and Technology, Coimbatore, India \\ ${ }^{a}$ E-mail address: janu_sathya@rediffmail.com
}

(received 28 June 2018; revised 20 November and 16 December 2018; accepted 14 March 2019)

\begin{abstract}
Diabetic Retinopathy (DR) is one of the leading causes of visual impairment. Diabetic Retinopathy is the most recent technique of identifying the intensity of acid secretion in the eye for diabetic patients. The identification of DR is performed by visual analysis of retinal images for exudates (fat deposits) and the main patterns are traced by ophthalmologists. This paper proposes a fully automated Computer Assisted Evaluation (CAE) system which comprises of a set of algorithms for exudates detection and to classify the different stages of Diabetics Retinopathy, which are identified as either normal or mild or moderate or severe. Experimental validation is performed on a real fundus retinal image database. The segmentation of exudates is achieved using fuzzy C-means clustering and entropy filtering. An optimal set obtained from the statistical textural features (GLCM and GLHM) is extracted from the segmented exudates for classifying the different stages of Diabetics Retinopathy. The different stages of Diabetic Retinopathy are classified using three classifiers such as Back Propagation Neural Network (BPN), Probabilistic Neural Network (PNN) and Support Vector Machine (SVM). The experimental results show that the SVM classifiers outperformed other classifiers for the examined fundus retinal image dataset. The results obtained confirm that with new a set of texture features, the proposed methodology provides better performance when compared to the other methods available in the literature. These results suggest that our proposed method in this paper can be useful as a diagnostic aid system for Diabetic Retinopathy.
\end{abstract}

Key words: diabetic retinopathy; fundus retinal images; exudates; fuzzy C-means; entropy filtering; disease classification.

\section{Introduction}

Diabetic Retinopathy (DR) is one of the most important causes of visual impairment. Exudates are one of the major signs for the existence of Diabetic Retinopathy, which arises due to leakage of fats and proteins as yellow lesions of various volumes on the eyes. The patient may suffer complete blindness if the exudates are not identified in advance which is due to the accumulation of exudates in the fundus oculus. Recurrent screening is required to identify an early stage of DR. Even though diabetes does not certainly involve vision loss, about $2 \%$ of the diabetic patients affected by DR are blind and $10 \%$ of the diabetic patients experience vision degradation after 15 years of diabetes as a result of DR complications. Diabetic patients with DR complications observe no symptoms until visual impairment develops. Eye fundus examination is necessary for diabetic patients every year to diagnose the problems that they can receive treatment on time [1].
The exudates generally form clusters that can be scattered all over the retina, which are easily perceptible in retina images and their existence indicates that the patient has DR [2]. If the exudates occur in the macula region and they can cause vision loss [3]. Moreover, early diagnosis and treatment of diabetic retinopathy may avoid or reduce the vision degeneration to the patients [3].

The diagnosis of DR is done by visual examination by an ophthalmologist or a trained professional. The accuracy of the diagnosis depends on factors such as image quality, skill, and experience of the specialist, among others. Thus, an automatic system for the diagnosis of diseases of the visual system, through retinal images, can reduce the workload of medical professionals [1].

The objective of our work is to develop a Computer Assisted Evaluation (CAE) system with a set of computer algorithms for automatic exudates segmentation and detection. An optimal set of statistical texture features are extracted from the segmented 
exudates. The retrieved features are fed as input to the classifier to categorize different stages of Diabetics Retinopathy as either normal or mild or moderate or severe which is vital for assessing the medical therapies and risk factors for this frequent complication of diabetes. The proposed CAE system will increase the effectiveness of preventive protocols and early therapeutic treatments. Furthermore, the system would result in benefits of quick identification of disorders present and also the identification of the severity stages of the disorders.

The rest of the paper is organized as follows: in section 2, we present the related works of automatic exudates detection and automatic retinal images classification. The proposed CAE system for exudates detection and analysis of different stages of DR is presented in section 3. Section 4 gives the empirical results and analysis, and we draw conclusions in section 5 .

\section{Related Works}

Up to this moment, exudates detection and classification from fundus retinal images is still very challenging. Different research works on the detection of exudates and classification of Diabetic Retinopathy stages in fundus retinal images has been proposed. Automatic detection of diabetic retinopathy and glaucoma through image processing and feature extraction of the entire fundus image and classification using data mining techniques is proposed in [4]. An improved model of classification using weighting techniques to select the best representative features for classifying retinal fundus image as the normal and severe image is presented in [5]. A new automatic segmentation system based on the morphological operation and thresholding is proposed in [6]. Computational Intelligence based on Fuzzy C Means clustering approach for detection of exudates and multilayer neural network classifier used for classification of exudates and non-exudates parameters in the fundus retinal images of the eye is proposed in [7]. Detection of blood vessels and exudates in color fundus images based on the new feature set is presented in [8]. Robust exudates detection and segmentation from color retinal images for mass screening of diabetic retinopathy is proposed in [9]. Identification of diabetic retinal exudates in digital colour images using support vector machine is proposed in [10]. ROIbased segmentation and morphological reconstruction for exudate detection are presented in [11]. The fundus images in the LAB color space using CLAHE is implemented to enhance its contrast for detection of exudates and classified as either "exudates" or "non-exudates" based on their high grey-level variations via employing an artificial neural network is proposed in [12]. The severity grade of diabetic retinopathy using a machine learning system is proposed in [13]. The orientation scores of the retinal image are used to obtain an orientation-enhanced image to detect the exudates is proposed in [14]. A deep convolution neural network for detection of exudates is discussed in [15]. In order to increase the performances of classifications, the voting scheme and NN approaches were combined in [16]. The new method for computer aided detection using Gabor filters and support vector machine classifier for diabetic retinopathy identification, developed in [17].

Among several exudates detection and risk level classification methods available, artificial intelligence techniques draw more attention from researchers for using it for exudates detection. Even though many significant efforts have been employed in developing efficient algorithms for exudates detection and risk level classification; there is quite much research work to be done.

\section{Proposed CAE System for Segmentation and Classification of Exudates Method}

The proposed CAE system involves four processing steps in the detection of disorders in the retinal fundus images is shown in Figure 1. Preprocessing step enhances the quality of the image for further processing steps. The second stage involves segmentation and detection of exudates. The statistical texture features are extracted from the segmented exudates in the third stage. In the fourth stage, the extracted features are used for Risk level Classification of DR in the fundus retinal image.

\subsection{Image Preprocessing}

Preprocessing aims at improving the quality of each input image and reducing the computational burden for subsequent analysis steps. In fundus retinal images the green band is largely used for identification of exudates, than red and blue bands because it gives more information. Morphological filtering is applied to the green channel image to remove vessel central light reflex, as it may contribute to false detection of exudates. The arithmetic mean kernel is used for background homogenization in order to obtain smooth intensity values uniformly.

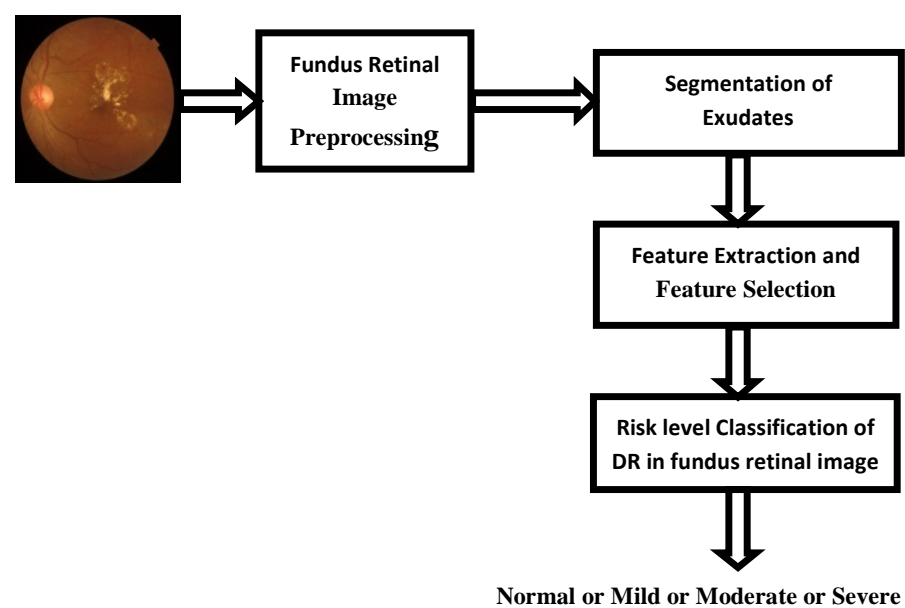

Figure 1. Block diagram of Proposed CAE System for Fundus Retinal Image Exudate Detection and risk level classification of Diabetic Retinopathy. 


\subsection{Segmentation of Exudates}

The exudates are extracted by eliminating blood vessels and optic disc from the green channel image. The steps involved in exudates detection are as follows.

\section{Step 1: Blood vessel segmentation}

During the segmentation of exudates, the blood vessels have a bright lesion like appearance. In order to improve the accuracy of exudates segmentation and to reduce false positive detection, blood vessels are removed. Fuzzy C-Means (FCM) clustering algorithm is used to segment the blood vessel. FCM is a data clustering technique where each data point belongs to a cluster of some degree that is specified by a membership grade. It is based on the minimization of the objective function given in Equation 1:

$J=\sum_{j=1}^{N} \sum_{i=1}^{c} u_{i j}^{m}\left\|x_{j}-v_{i}\right\|^{2}$

$$
1 \leq \mathrm{m} \leq \infty \quad \text { Eq. } 1
$$

where $\mathrm{m}$ is any real number greater than $1, u_{\mathrm{ij}}$ is the degree of membership of $x_{i}$ in the cluster $j, x_{i}$ is the $i^{\text {th }}$ measured data, $v_{j}$ is the centre of the cluster, and $\left\|^{*}\right\|$ is any norm expressing the similarity between any measured data and the cluster centre. The parameter $m$ controls the fuzziness of the resulting partition and $m=2$ is used in this work.

The cost function is minimized when pixels close to the centroid of their clusters are assigned high membership values and low membership values are assigned to pixels with data far away from the centroid. The value of the membership function represents the probability of a pixel belonging to a particular cluster. In the FCM algorithm, the probability is dependent solely on the distance between the pixel and each individual cluster centroid in the feature domain. The value of membership functions and cluster centers are updated by the following Equations 2 and 3.

$u_{i j}=\frac{1}{\sum_{k=1}^{c}\left(\frac{\left\|x_{j}-v_{i}\right\|}{\left\|x_{j}-v_{k}\right\|}\right)^{2 /(m-1)}}$

and

$v_{i}=\frac{\sum_{j=1}^{N} u_{i j}^{m} x_{j}}{\sum_{j=1}^{N} u_{i j}^{m}}$

Starting with an initial guess for each cluster center, the FCM algorithm tends to converge to a solution for representing the local minimum or a saddle point of the cost function. Convergence can be identified by comparing the changes in the membership function or the cluster center at two successive iteration steps.

\section{Step 2: Optical disc segmentation}

The segmentation of optic disc is crucial since it is circular in shape with high contrast and is similar to exudates. A circular mask is used for removing the optic disc. Increase in size of optic disc leads to Glaucoma.

\section{Step 3: Exudate segmentation}

The detection and quantification of exudates will contribute to the mass screening and assessing of the diabetic retinopathy. Exudates detection without user interaction is not a simple task, because there is no prior knowledge about its presence or its distribution. The size, shape, color, and thickness vary from one patient to another patient. The exudates abnormality is detected using entropy filtering. By employing entropy filtering over the preprocessed image, the filtered output clearly segments blood vessels, optic disc, and exudates. The blood vessels segmented in step 1 and the optic disc obtained in step 2 are subtracted from the entropy filtered output image to extract the exudates separately.

\subsection{Feature Extraction}

Once the exudates are identified, it is necessary to identify the risk level of Diabetic Retinopathy, i.e., whether the Diabetic Retinopathy is normal or mild or moderate or severe. For quantitative evaluation, many features have been retrieved from the exudates of the fundus retinal images extracted during segmentation. For retinal image exudates, risk level classification statistical texture features are widely used [3$8,10,12-17]$. In this proposed CAE system, a set of 14 GLCM textural features and 4 GLHM (gray level histogram moments) features as defined in [18-21] were extracted from the exudates. These features are used because they have proven an improvement in classifier performance [4,5,7,10,12-17]. The features extracted are: entropy, skewness, difference moment, energy measure, difference variance, sum average, standard deviation, difference average, difference entropy, kurtosis, correlation, inertia, variance, inverse difference moment, mean, sum entropy, sum variance and information measure of correlation.

\subsection{Feature Selection}

Feature selection has been widely used to improve the prediction accuracy of classifiers. The improvement in prediction is related to the removal of redundant features or noisy features in the data which can be eliminated by feature selection, relatively few features used in a classifier can keep the classification performance robust. Hypothesis tests appear to be well suited for dealing with the selection of most discriminative feature subset from the available feature set [2225]. The test indicates that only six features can discriminate between the different risk levels they are: correlation, cluster shade, dissimilarity, kurtosis, mean and entropy. These selected features are not used in other classifiers that were discussed in the literature. The feature extraction and selection are a key step in risk level classification since the performance of CAE depends on both the optimization of the feature selection and the classification method. 


\section{Correlation}

Correlation is the grey level linear dependence between the pixels at a specified position to each other as in Equation 4:

Correlation $=\frac{\sum_{i} \sum_{j}(i, j) p(i, j)-\mu_{x} \mu_{y}}{\sigma_{x} \sigma_{y}}$

Where $p(i, j)$ is the pixel value at the point $(i, j)$ of an image, $\mu$ and $\sigma$ are the mean and standard deviation respectively.

\section{Cluster shade}

Cluster shade is a measure of the skewness of the matrix or lack of symmetry. When the value of cluster shade is higher, the image is not symmetric with respect to the texture value. The cluster shade is estimated using Equation 5:

Cluster shade $=\sum_{i, j}^{i, j}\left(\left(i-\mu_{i}\right)+\left(j+\mu_{j}\right)\right)^{3} c(i, j)$

$\mathrm{C}(\mathrm{i}, \mathrm{j})$ - is the $(\mathrm{i}, \mathrm{j})$ the entry in co-occurrence matrix $\mathrm{C}$

$\sum_{i}$ means $\sum_{i=1}^{i=M}$ where $\mathrm{M}$ is the number of rows

$\sum_{j}$ means $\sum_{j=1}^{j=N}$ where $\mathrm{N}$ is the number of columns

$\sum_{i, j}$ means $\sum_{i}, \sum_{j}$

$\mu_{i}$ is defined as $\mu_{i}=\sum_{i} i \sum_{j} c(i, j)$

$\mu_{j}$ is defined as $\mu_{j}=\sum_{i} j \sum_{j} c(i, j)$

\section{Dissimilarity}

It is a measure that defines the variation of grey level pairs in an image. It is computed as in Equation 6:

Dissimilarity $=\sum_{i, j}|i-j| p(i, j)$

Where $p(i, j)$ is the pixel value at the point $(i, j)$ of an image.

\section{Mean Value}

The mean, $\mu$ of the pixel values in the defined window, estimate the value in the image in which central clustering occurs, calculated using Equation (7).

$\mu=\frac{1}{M N} \sum_{i=1}^{M} \sum_{j=1}^{N} p(i, j)$

Where $p(i, j)$ is the pixel value at point $(i, j)$ of an image.

\section{Kurtosis}

The Kurtosis, $\mathrm{K}$ measures the peakness or flatness of distribution relative to a normal distribution. The conventional definition of kurtosis is given in Equation 8.

$K=\left\{\frac{1}{M N} \sum_{i=1}^{M} \sum_{j=1}^{N}\left[\frac{p(i, j)-\mu}{\sigma}\right]^{4}\right\}-3$

Where, $p(i, j)$ is the pixel value at point $(i, j), \mu$ and $\sigma$ are the mean and standard deviation respectively. The -3 term makes the value zero for a normal distribution.

\section{Entropy}

Entropy shows the amount of information of the image that is needed for image compression. Entropy measures the loss of information in a transmitted image as in Equation 9.

Entropy $=-\sum_{i, j} p(i, j) * \log (p(i, j))$

Eq. 9

\subsection{Risk Level Classification}

Once a proper representation in a feature space has been chosen for the patterns, a classifier should be trained and tested on the data. The role of the classifier is to implement a decision rule that will indicate to which class a given pattern belongs. Some efforts have already been made to automatically predict the risk level [3-8,10,12-17]. Risk level classification algorithms are in continuous development and improvement.

\section{Investigational Analysis of Risk Level Classifier Models}

The classification methods are used to classify the detected exudates in the fundus retinal images into Normal or Mild or Moderate or and Severe Diabetics Retinopathy. The selection of the correct classifier is a factor that very sensitively affects the performance of the correct classification. The selected classifier needs to fit well to the training data (i.e. produce a good separation between the classes) while, on the other hand, being as robust as possible to unseen data (generalizability). In this proposed work, three types of classifiers such as Back Propagation Network (BPN), Support Vector Machine (SVM) and Probabilistic Neural Network (PNN) were evaluated for classification of the different stages of Diabetics Retinopathy of detected exudates in the fundus retinal images and to find an optimal classifier that outperforms all other explored classifiers for the features extracted and the examined dataset of fundus retinal images.

\section{Artificial Neural Network (ANN) Classifier}

The proposed artificial neural network classifier model implemented includes a three-layered network topology - it has an input layer, an output layer, and a hidden layer. The number of nodes present in the input corresponds to the number of input variables, which are six in this experiment because six texture features are selected for classification. The output layer contains one node with values from zero to one indicating the different stages of diabetic retinopathy; the hidden layer contains five nodes. The proposed artificial neural network classifier model implemented includes a three-layered network topology - it has an input layer, an output layer, and a hidden layer. The mean-square difference is used to estimate the error. The learning coefficient of 0.0001 is set for the hidden layer and 0.01 for the output layer. The linear activation function is used for the hidden layer and the hyperbolic tangent transfer function is considered as activation functions for the output layer.

\section{Back Propagation Algorithm}

The back propagation learning algorithm is a supervised learning method that can be used with multilayer networks and nonlinear differentiable transfer functions [25]. The power of the back propagation algorithm is that it allows us to compute an effective error value for each node in the hidden unit and thus adjust the hidden weights. The back propagation learning algorithm consists of presenting an input pattern and updating the network connection weights to bring the actual outputs 
closer to the desired target values. These actual outputs are compared to the target values; any difference corresponds to an error. This error is some scalar function of the weights, thus the weights are adjusted to reduce the error. This error function is the sum of squared differences of the actual outputs and targets. Let $\mathrm{V}_{\mathrm{j}}$ be the training set elements, $\mathrm{Y}_{\mathrm{j}}$ are the outputs and $\mathrm{O}_{\mathrm{j}}$ are the desired output or targets, then the error function of the $\mathrm{i}^{\text {th }}$ pattern is shown in Equation 10.

$E=\sum_{i} \sum_{j}\left(y_{j}^{i}-o_{j}^{i}\right)^{2}$

The back propagation weight adjustment is done through Equation 11.

$w_{i j}(k+1)=w_{i j}(k)-\eta \frac{\partial E}{\partial w_{i j}}$

Where $\mathrm{k}$ is the iteration number and $\eta$ is the learning rate. The weights are adjusted continuously until a "better performance" is reached.

The back propagation neural network has a high success rate in elucidating many complex problems, but it still has some drawbacks. If the learning rate is small, the training process or learning process is stable but at the expense of computation time. If the network topology is not carefully selected, the back propagation algorithm can get easily trapped in local minima or it might lead to slow convergence or even network failure.

\section{Support Vector Machine (SVM) Classifier}

ANNs have proven to be good classifiers, but they require a large number of samples for training, which is not always true in practice. Support vector machines (SVMs) are based on statistical learning theory and they specialize for a smaller sample number. The SVMs have better generalization than ANNs and guarantee the local and global optimal solution similar to that obtained by ANN [26].

Support vector machines (SVMs) are an emerging area of investigation in machine learning and pattern recognition. SVMs are a machine learning method for creating a classification function from a set of labeled training data. The basic concept of SVM is to build a hyperplane as the decision surface in such a way that the boundary of separation among positive and negative examples is maximized [10].

The input training vectors $x_{i}$ and $x_{j}$ (set of input feature vector and predicts) are mapped into a higher dimensional space by a function $\varnothing$. Then SVM constructs a linear separating hyperplane with the maximal margin in this higher dimensional space. Furthermore, $\mathrm{K}\left(\mathrm{x}_{\mathrm{i}}, \mathrm{x}_{\mathrm{j}}\right)=\varnothing\left(\mathrm{x}_{\mathrm{i}}\right) \times \varnothing\left(\mathrm{x}_{\mathrm{j}}\right)$ is called the kernel functions [10]. For this experimental work, the linear kernel is used. The linear kernel function is given by

\section{Equation 12.}

$$
\mathrm{K}\left(x_{\mathrm{i}}, x_{j}\right)=x_{i} \bullet x_{j}
$$

Where $x_{i}$ and $x_{j}$ are input vectors comprised of one of the previously mentioned feature vectors and predicts, $\bullet$ is the dot product operation.
SVMs have been shown to perform well in medical diagnosis applications and have also been shown to perform well when dealing with relatively small training sets [10]. This is particularly appealing given the inherent difficulty in acquiring large amounts of screening data devoted exclusively to training. The support vector machines also perform well and classify reasonably quickly on high dimensional data [10].

\section{Probabilistic Neural Network (PNN)}

A Probabilistic Neural Network (PNN) is a feed-forward neural network widely used in classification and pattern recognition problems. In the PNN algorithm, the Parent Probability Distribution Function (PDF) of each class is approximated by a Parzen window and a non-parametric function. PNN classifier maps any input pattern to a number of classifications. So the probability of miss-classification is minimized [27].

There are three layers in architecture - the first layer is the input layer, second is the pattern layer, and the third is the summation layer. When an input pattern is presented, the input layer computes distances from the input vector to the target vectors and generates a vector whose elements specify how close the input is to a target vector. These contributions for each class of inputs are summed up in the pattern layer to produce as its net output a vector of probabilities. Finally, the summation layer picks the maximum of these probabilities [27].

The total neurons in Pattern Layer are based on their corresponding class. If there are $\mathrm{C}$ Classes and $\mathrm{n}$ Neurons, then in each group there will be $n / C$ neurons. Output $X$ at each neuron will be computed by a probability density function (generally used Gaussian distribution) given in Equation 13.

$g_{i}(x)=\frac{1}{\sqrt{2 \Pi \sigma^{2}}} \exp \left\{-\left(\left(\left\|X-x_{j}\right\|\right)^{2} / 2 \sigma^{2}\right)\right\}$

Where $\mathrm{i}=1 \ldots \mathrm{n}$ (Neurons in Pattern Layer); $\mathrm{j}=1 \ldots \mathrm{k}$ (Number of Inputs in Input Layer) is the smoothing parameter ( values depends on the data $\sigma$ set or estimated heuristically).

In summation layer, all the neurons which belong to that class will be summed up here as given in Equation 14.

$f_{i}(x)=\sum_{j=1}^{l} g_{l}(x)$

Eq. 14

where $\mathrm{i}=1 \ldots \mathrm{C}$ (Classes); 1 is the number of neurons which belongs to that class.

The Output Layer decides in which class test sample belongs by comparing the f's values of the pattern layer using Equation 15.

If $f_{i}(x) \geq f_{j}(x), / /$ Given $\mathrm{i} \neq \mathrm{j}$

Then $x \in i$ ( $\mathrm{e}^{\text {th }}$ number of classes)

Else

$$
x \in j\left(\mathrm{e}^{\text {th }} \text { number of classes }\right)
$$

End 
Probabilistic Neural Network (PNN) guarantees to provide optimal classification as the size of the representative training set increases. The training patterns can be added or removed without extensive retraining. But the major difficulty is to find an accurate smoothing parameter $\sigma$.

\section{Experimental Results and Discussion}

This section presents the visual and statistical experimental results and analysis of the proposed computer assisted evaluation system for exudates detection and risk level classification of diabetic retinopathy on real fundus retinal image dataset.

\subsection{Evaluation Data and Methods}

The fundus retinal image dataset contains records of 160 patients, which are taken using the fundus camera TRC-50DX. The fundus retinal image dataset along with the ground truth is received from the Aravind Eye Hospital, Coimbatore, India.

The test fundus retinal images are pre-processed to obtain a green plane image which enhances the quality of the retinal image and makes the analysis easier. From the green plane of the image, the blood vessels are segmented separately in order to find the presence of exudates parameters in the retinal test image is shown in Figure 2. Finally, the segmented exudates are detected and converted into RGB image with green patches on the exudate part of the retinal image makes easier identification of the presence of exudates as shown in

Figure 3 .
The exudate regions of the retinal fundus images for Normal, Mild, Moderate and Severe DR are overlaid on the original input retinal images for human perception. The overlaid retinal input images are listed in Figure 3. Exudate patches of Normal, Mild level of DR, Moderate level of DR, Severe level of DR on the retinal fundus images are shown in Figure 3.

The retinal images of the Mild DR patients will have a few exudate patches overlaid laid as green patches in their original retinal image. Similarly, the retinal images of the Mild DR will have more exudate patches than the Mild DR overlaid on the original retinal image. The retinal images of the Severe DR patients will more exudate patches on the retinal images overlaid when compared to the Mild DR and Mild DR patients.

\subsection{Performance Evaluation}

Three classifiers are designed and their structure is individually optimized such that the performances of the individual classifiers are maximized. The performance of the classifier model used in the proposed CAE system is validated using cross-validation experimentation, for cross-validation the fundus retinal image dataset is divided into the training set and the test set. The training set of 100 images from the dataset is used to build the classifier model and the testing set of 60 images from the dataset is used to evaluate the trained classifier model. The training dataset images are not used to testing the classifier model.

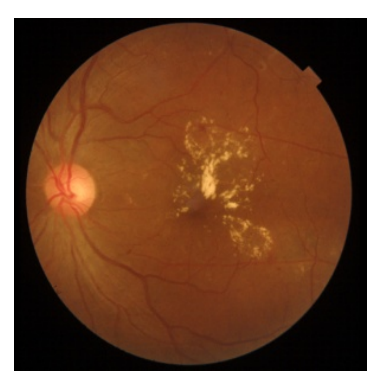

(a) Test image

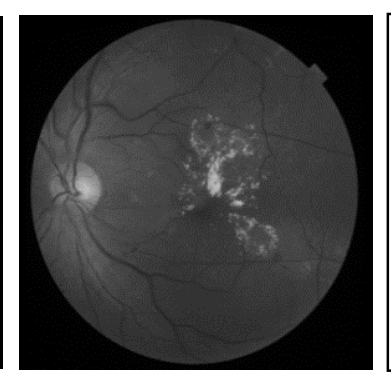

(b) Green plane

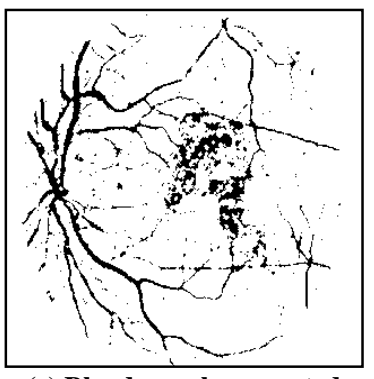

(c) Blood vessel segmented image

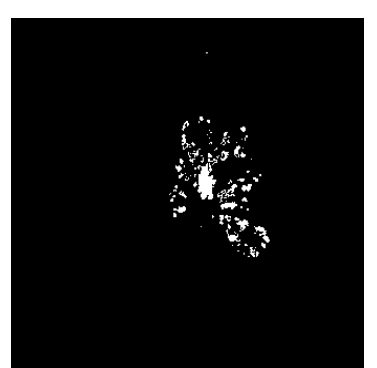

(d) Exudate Segmentation

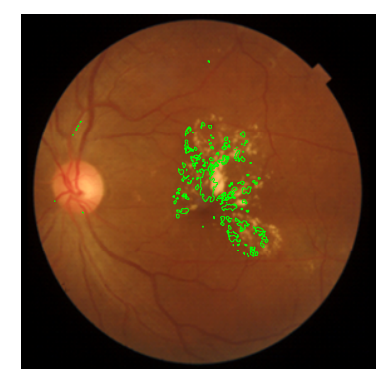

(e) Exudate Detection

Figure 2. Exudate Detection.

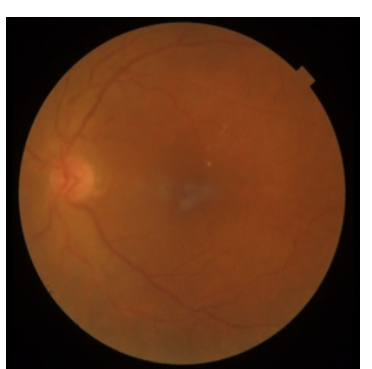

Normal Retinal image

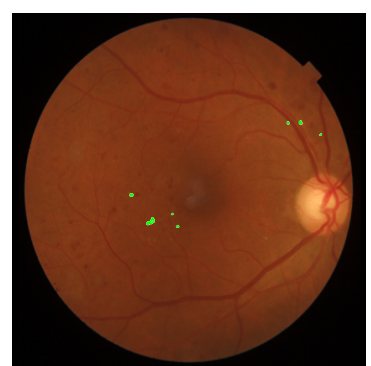

Mild DR

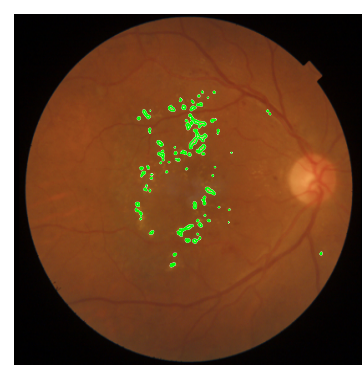

Moderate DR

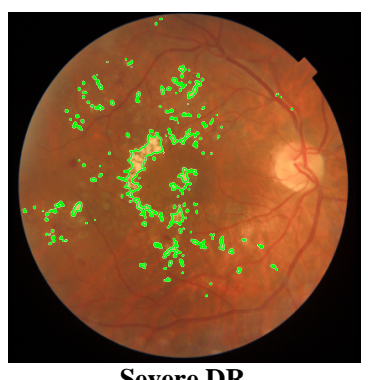

Severe DR

Figure 3. The exudate regions of the retinal fundus images for Normal, Mild, Moderate and Severe DR are overlaid on the original input retinal. 
The classification performance can be assessed in terms of the sensitivity, specificity, accuracy, and Error in the classification of the system as derived from the Equations 16-19.

$$
\begin{aligned}
& \text { Accuracy }(A C C): A C C=\frac{T P+T N}{T P+F P+F N+T N} \\
& \text { Specificity }(S P): S P=\frac{T N}{T N+F P} \\
& \text { Sensitivity: } S N=\frac{T P}{T P+F N}
\end{aligned}
$$

Error in classification (E): $E=1-$ Accuracy

Where $\mathrm{TP}=$ true positive, $\mathrm{TN}=$ true negative, $\mathrm{FP}=$ false positive, and $\mathrm{FN}=$ false negative. It is clear that the main purpose of any classifiers to reduce the false positive and negative rates, similarly, to exploit maximum true negative and positive rates.

It is obvious that the main objective of a classifier is to maximize the true negative and positive rates or, similarly, to minimize the false positive and negative rates. The sensitivity, specificity, accuracy, and error of the classification technique were evaluated through quantitative measures derived through the comparison of each classified result with its corresponding ground truth. Ground truth is based on the diagnosis of the Ophthalmologist, who analyses the fundus retinal images.

The comparison of classifiers is made in order to determine and signify the best classifier for the provided dataset of real fundus retinal images. The results obtained by the proposed three classifier models for CAE system using cross-validation method are presented in Table 1. The results indicate that the selected texture features for classifier provide better discrimination between various risk stages of diabetic retinopathy than other methods that were proposed in the literature. The results of the experiments also imply that combining different types of statistical texture information (GLCM and GLHM) into a single feature set may improve the discriminatory power of a feature set without increasing its size.

The comparison of classifiers is made in order to determine and signify the best classifier for the provided dataset of real fundus retinal images. The results obtained by the proposed three classifier models for CAE system using cross-validation method are presented in Table 1. The results indicate that the selected texture features for classifier provide better discrimination between various risk stages of diabetic retinopathy than other methods that were proposed in the literature. The results of the experiments also imply that combining different types of statistical texture information (GLCM and GLHM) into a single feature set may improve the discriminatory power of a feature set without increasing its size.

The overall results of performance evaluation obtained from classifiers indicate that the optimal feature set which has been extracted by statistical t-test provides high accuracy, sensitivity, and high specificity than the available methods reported in the literature. This will lead to a natural development of a CAE system capable of assisting health professionals in the painstaking task of tracing fundus retinal image in search of abnormalities.

\section{Proposed CAE System Snapshots}

The proposed CAE system is developed as an Android-based mobile application and it is shown in Figure 4.

\section{Conclusion}

This paper introduced a set of methods for processing and analyzing fundus retinal images. An efficient segmentation method along with best-performing texture features and classifiers for risk level classification of diabetic retinopathy on real Fundus Retinal Image dataset. Fuzzy C-means and entropy filtering based exudates segmentation is used for efficient detection of exudates. A set of 18 textural features (GLCM and GLHM) information are extracted from the exudates of the fundus retinal image dataset. The features subset is selected using statistical hypothesis t-test, which results in indicating six features which are best in discriminating between various risk

\begin{tabular}{|c|c|c|c|}
\hline ClassifierType & Artificial Neural Network (ANN) & Probabilistic Neural Network (PNN) & "Support Vector Machine (SVM) \\
\hline Number of cases used for classification & 60 & 60 & 60 \\
\hline True positive & 37 & 34 & 39 \\
\hline True negative & 17 & 16 & 19 \\
\hline False positive & 3 & 4 & 1 \\
\hline False negative & 3 & 6 & 1 \\
\hline Sensitivity (\%) & 92.5 & 85 & 97.5 \\
\hline Specificity (\%) & 85 & 80 & 95 \\
\hline Accuracy $(\%)$ & 90 & 83.33 & 96.67 \\
\hline Error in classification (\%) & 10 & 16.67 & 3.33 \\
\hline No of cases misdiagnosed & 6 & 10 & 2 \\
\hline
\end{tabular}
levels of Diabetic Retinopathy.

Table 1. Detailed investigation of the classifiers proposed for Diabetic Retinopathy stage classification. 


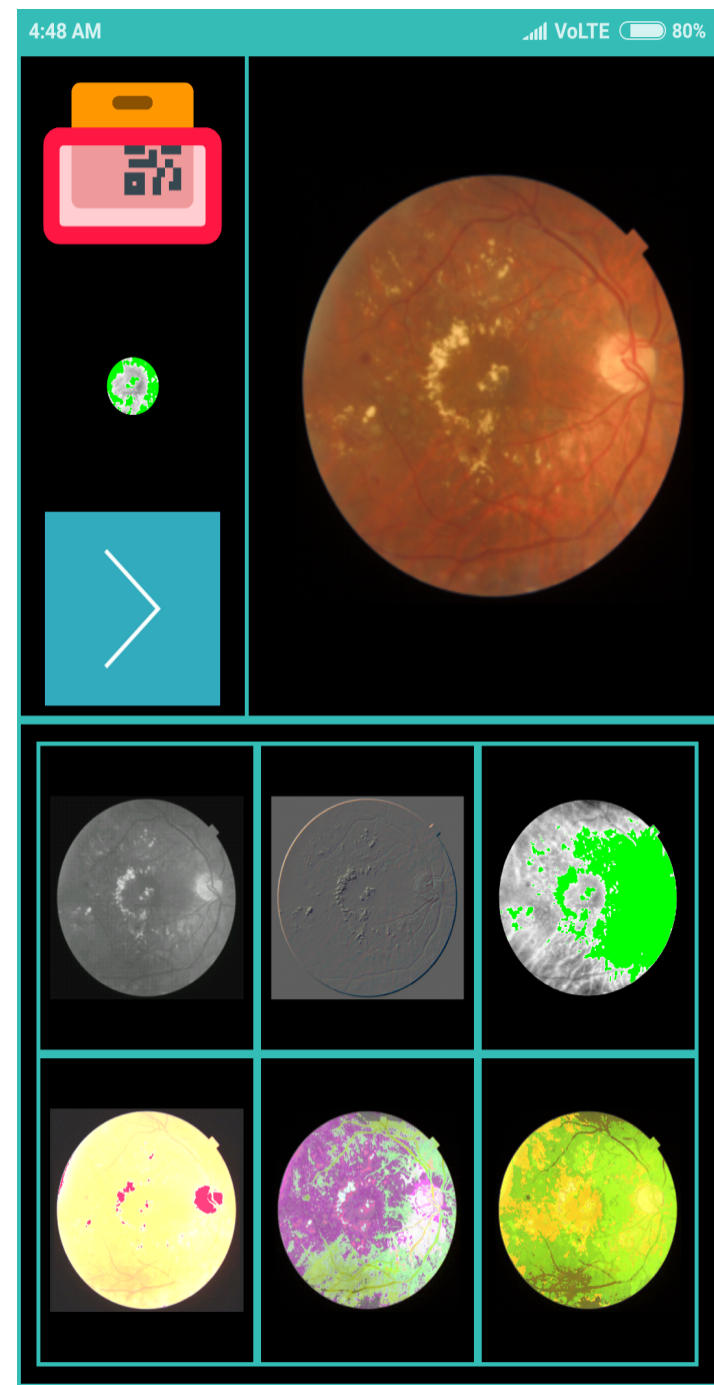

(a) Feature Extraction

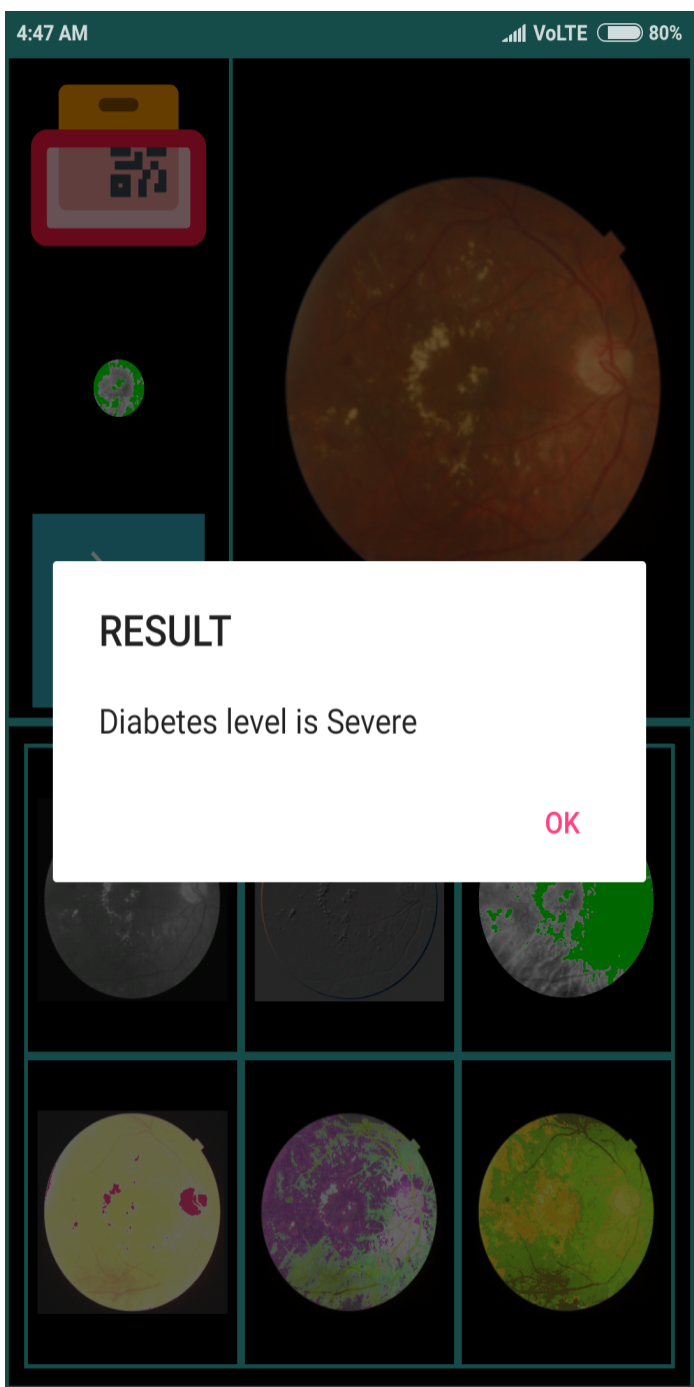

(b) Risk Level Classification

Figure 4. Snapshots of Android-based mobile application of proposed CAE system.

Three types of classifiers such as Support Vector Machine (SVM), Back Propagation Network (BPN) and Probabilistic Neural Network (PNN) were analyzed for classification of the different stages of Diabetics Retinopathy of detected exudates in the fundus retinal images and it is found that SVM classifier outperforms all other explored classifiers for the features extracted and the examined dataset of fundus retinal images. The proposed set of methods can be used as part of a larger CAE system to improve diabetic retinopathy diagnosis.

\section{Acknowledgement}

We thank Aravind Eye Hospital, Coimbatore, Tamil Nadu, India, for providing the fundus retinal images dataset along with its ground truth results used for validating the proposed CAE system.

\section{References}

[1] Lee N, Laine AF, Smith RT. A hybrid segmentation approach for geographic atrophy in fundus auto-fluorescence images for diagnosis of age-related macular degeneration. In Engineering in Medicine and Biology Society, 2007. EMBS 2007. 29th Annual International Conference of the IEEE, pp. 4965-4968. IEEE, 2007.

[2] Sopharak A, Dailey NM, Uyyanonvara B, et al. Machine learning approach to automatic exudate detection in retinal images from diabetic patients. Journal of Modern Optics. 2010;57(2):124-135.

[3] Giancardo L, Meriaudeau F, Karnowski TP, et al. Exudate-based diabetic macular edema detection in fundus images using publicly available datasets. Medical Image Analysis. 2012;16(1):216-226. 
[4] Geetha Ramani R, Balasubramanian L, Jacob SG. Automatic Prediction of Diabetic Retinopathy and Glaucoma through Retinal Image Analysis and Data Mining Techniques. 2012 International Conference on Machine Vision and Image Processing (MVIP), Taipei, 2012, pp. 149-152. IEEE, 201.

[5] Wagle S, Mangai JA, Kumar VS. An Improved Medical Image Classification Model using Data Mining Techniques. GCC Conference and exhibition, November 17-20, Doha, Qatar. IEEE, 2013.

[6] Hatanaka Y, Muramatsu C, Sawada A, et al. Glaucoma Risk Assessment Based on Clinical Data and Automated Nerve Fiber Layer Defects Detection. 34th Annual International Conference of the IEEE EMBS San Diego, California USA, 28 August - 1 September, 2012.

[7] Osareh A, Shadgar B, Markha R. A Computational Intelligence Based Approach for Detection of Exudates in Diabetic Retinopathy Images. IEEE Transactions on Information Technology in Biomedicine, 2009;13(4):535-545.

[8] Youssef D, Solouma N, El-dib A, et al. New Feature-Based Detection of Blood Vessels and Exudates in Color Fundus Images. 2010 2nd International Conference Image Processing Theory Tools and Applications (IPTA), Paris. pp. 294-299. 2010.

[9] Zhang X, Thibault G, Decenciere E, et al. A. Exudate Detection in Color Retinal Images for Mass Screening of Diabetic Retinopathy. Medical Image Analysis. 2014;18(7):1026-1043.

[10] Mansour RF, Abdelrahim EM, Al-Johani AS. Identification of Diabetic Retinal Exudates in Digital Color Images Using Support Vector Machine. Journal of Intelligent Learning Systems and Applications. 2013;5:135-142.

[11] Sreng S, Takada J, Maneerat N, et al. Automatic exudate extraction for early detection of diabetic retinopathy. Proceedings of the International Conference on Information Technology and Electrical Engineering, Oct. 7-8, IEEE Xplore Press, Yogyakarta, pp: $31-$ 35, 2013.

[12] Franklin SW, Rajan SE. Diagnosis of diabetic retinopathy by employing image processing technique to detect exudates in retinal images. IET Image Processing. 2014;8(1):601-609.

[13] Roychowdhury S, Koozekanani DD, Parhi KK. DREAM: Diabetic retinopathy analysis using machine learning. IEEE J Biomed Health Informat. 2014;18(5):1717-1728

[14] Liu Q, Zou B, Chen J, et al. A location-to-segmentation strategy for automatic exudate segmentation in colour retinal fundus images. Comput Med Imag Graph. 2017;55:78-86.

[15] Caramihale T, Popescu D, Ichim L. Interconnected neural networks based on voting scheme and localdetectors for retinal image analysis and diagnosis. In Proceedings of the Image Analysis and Processing Conference - ICIAP 2017, Catania, Italy, Battiato, 1115 September 2017; Gallo G, Schettini R, Stanco F (Eds). Lecture Notes in Computer Science. 2017;10485:753-764.

[16] Raja SS, Vasuki S. Screening diabetic retinopathy in developing countries using retinal images. Appl Med Inform. 2015;36(1):13-22.

[17] Sathya J, Geetha K. Automatic segmentation of lesion from breast DCE-MR image using artificial fish swarm optimization algorithm. Pol J Med Phys Eng. 2017;23(2):29-36.

[18] Gibbs P, Turnbull LW. Textural analysis of contrast enhanced MR images of the breast. Magn Reson Med. 2003;50(1):92-98.

[19] Agner SC, Soman S, Libfeld E, et al. Textural kinetics: a novel dynamic contrast-enhanced (DCE)-MRI feature for breast lesion classification. J Digit Imaging. 2011;24(3):446-463.

[20] Haralick RM, Shanmugam K. Textural features for image classification. IEEE Transactions on Systems, Man, and Cybernetics. 1973;3(6):610-621.

[21] Vickers AJ. Parametric versus non-parametric statistics in the analysis of randomized trials with non-normally distributed data. BMC Med Res Methodol. 2005;35(5):1-12.

[22] Haury AC, Gestraud P, Vert JP. The influence of feature selection methods on accuracy, stability and interpretability of molecular signatures. PloS One. 2011;6(12):e28210.

[23] Foody GM. Classification accuracy comparison: hypothesis tests and the use of confidence intervals in evaluations of difference, equivalence and non-inferiority. Remote Sensing of Environment. 2009;113(8):1658-1663.

[24] Liu H, Li J, Wong L. A comparative study on feature selection and classification methods using gene expression profiles and proteomic patterns. Genome Inform. 2002;13:51-60.

[25] Lisboa PJ. A review of evidence of health benefits from artificial neural networks in medical intervention. Neural Networks. 2002;15(1):11-39.

[26] Levman J, Leung T, Causer P, et al. Classification of dynamic contrast-enhanced magnetic resonance breast lesions by support vector machines. IEEE Transaction on Medical Imaging. 2008;27(5):688-696.

[27] Mahendran G, Dhanasekaran R, Narmadha Devi KN. Identification of Exudates for Diabetic Retinopathy based on Morphological Process and PNN Classifier. International Conference on Communication and Signal Processing, April 3-5, 2014, India 